\title{
Comparación de las estrategias de campaña online para albergar la Agencia Europea de Medicamentos
}

\section{Comparing online campaigning strategies to host the European Medicines Agency}

\author{
Jorge Tuñón-Navarro; Daniel Catalán-Matamoros
}

Cómo citar este artículo:

Tuñón-Navarro, Jorge; Catalán-Matamoros, Daniel (2020). “Comparación de las estrategias de campaña online para albergar la Agencia Europea de Medicamentos". El profesional de la información, v. 29, n. 2, e290225.

https://doi.org/10.3145/epi.2020.mar.25

Artículo recibido el 09-04-2019

Aceptación definitiva: 10-12-2019

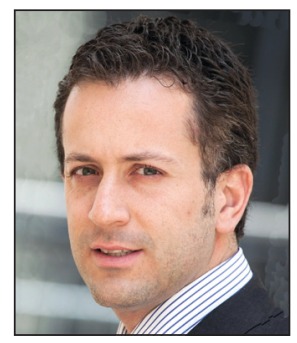

Jorge Tuñón-Navarro $ه$ https://orcid.org/0000-0003-0393-6560

Universidad Carlos III de Madrid Departamento de Comunicación Madrid, 126.

28903 Getafe (Madrid), España jorge.tunon.navarro@gmail.com

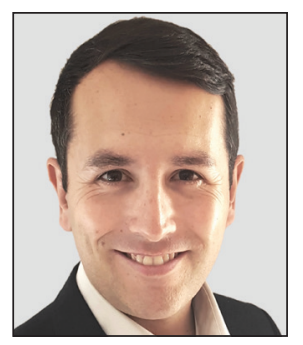

Daniel Catalán-Matamoros https://orcid.org/0000-0002-3086-6812

Universidad Carlos III de Madrid Departamento de Comunicación Madrid, 126.

28903 Getafe (Madrid), España dacatala@hum.uc3m.es

\section{Resumen}

Entre los diversos efectos secundarios del Brexit, la Comisión Europea reubicó la Agencia Europea de Medicamentos en Amsterdam. Las ciudades candidatas diseñaron diferentes campañas de comunicación política/institucional para convencer a los países miembros a su favor. Esta investigación compara estas campañas políticas/organizacionales online y destaca las temáticas y variables en cuestión durante el proceso de selección. A través de un análisis combinado de contenido preferentemente cuantitativo, el estudio pretende validar la medida en que las teorías resource push o resource pull aplicadas a las compañas organizacionales, tuvieron correlación con la decisión final de reubicar la agencia en Amsterdam.

\section{Palabras clave}

Brexit; Unión Europea; Comisión Europea; Comunicación política; Estrategias; Campañas online; Agencia Europea de Medicamentos; Comunicación organizacional; Comunicación digital.

\section{Agradecimientos}

Los autores de este artículo agradecen las críticas constructivas a una primera versión de este trabajo de investigación, proporcionadas tanto por un revisor anónimo como por Uxía Carral, doctoranda del Departamento de Comunicación de la Universidad Carlos III de Madrid.

\section{Financiación}

La presente investigación forma parte de tres proyectos europeos en vigor financiados por la Agencia Europea de la Educación, la Cultura y el Audiovisual (Eacea) de la Comisión Europea, el módulo Jean Monnet EuCofinanciado por el programa Erasmus+ de la Unión Europea

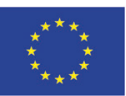
copol (referencia: 587167-EPP-1-2017-1-ES-EPPJMO-MODULE); la Red Jean Monnet Openeudebate (referencia: 600465-EPP-1-2018-1-ES-EPPJMO-NETWORK); y la Cátedra Jean Monnet Eudfake (referencia: 610538-EPP-1-2019-1-ES-EPPJMO-CHAIR).

El contenido de este artículo es responsabilidad exclusiva de los autores y la Eacea no es responsable del uso que pueda hacerse de la información aquí difundida.

Asimismo, este artículo es un resultado del Proyecto Fakenews financiado por la Agencia Española de Investigación del Ministerio de Ciencia, Innovación y Universidades del Gobierno de España (Referencia: RTI2018-097709-B-I00). 


\begin{abstract}
Among the diverse side effects of Brexit, the European Commission relocated the European Medicines Agency (EMA) to Amsterdam. Host bids designed different political/institutional communication campaigns to persuade member states in their favor. This investigation compares and contrasts these institutional political online campaigns and highlights the themes and variables under discussion during the selection process. Through a preferred quantitative content analysis, the aims to validate the extent to which resource push or resource pull theories are correlated with the final decision to relocate the Agency to Amsterdam.
\end{abstract}

\title{
Keywords
}

Brexit; European Union; European Commission; Political communication; Online campaigning; Strategies; European Medicines Agency; EMA; Organizational communication; Digital communication.

\section{Introducción}

Uno de los primeros efectos colaterales del Brexit tuvo lugar el 20 de noviembre de 2017, cuando la Unión Europea (UE) decidió reubicar la Agencia Europea de Medicamentos (EMA, según su acrónimo en inglés) de Londres a Amsterdam. Dado que albergar la EMA parecía atractivo, tanto en términos económicos como de branding, 19 ciudades de la UE candidatas compitieron para ser elegidas. Las autoridades de la UE (Unión Europea, 2017) diseñaron un procedimiento de cinco meses con votaciones finales al estilo Eurovisión. No sólo se pidió a los candidatos que presentaran una propuesta técnica que cumpliera los requisitos de selección, sino atraer (políticamente) y convencer a los demás estados miembros, para lo que llevaron a cabo una intensa campaña de comunicación organizacional/institucional y branding gubernamental.

Esta investigación se ha enfocado en torno al branding online a través de los portales web ad hoc de los candidatos, ha comparado y contrastado estas campañas online institucionales y políticas y ha destacado los temas y las variables decisivas durante el proceso de selección. El análisis ilustra cómo y por qué las estrategias de los candidatos difirieron en términos de agenda setting o selección temática (Semetko; De-Vresse, 2004) y extensión. A través de un análisis combinado de contenido eminentemente cuantitativo, esta investigación pretende validar la medida en que las teorías resource pull o resource push (las capacidades y los recursos económicos incentivan la involucración en campañas competitivas o por el contrario la ausencia de dichas capacidades es la que potencia esa competencia para la obtención de dichos recursos económicos) en comunicación organizacional están vinculadas con la decisión final de reubicar la agencia en Amsterdam.

En resumen, este artículo analiza las campañas online de los primeros 19 (y finalmente 16) candidatos para albergar la $E M A$, durante el proceso de selección. Se extraen conclusiones mediante una base de datos de textos, vídeos, fotografías, mapas, infografías, dibujos, tablas y gráficos. Además, tras establecer las preguntas de investigación basándose en otras campañas online institucionales, el artículo analiza las candidatas en función de varios factores, incluyendo contenido, encuadre y engagement respecto a las audiencias seleccionadas. Finalmente, concluirá con algunas observaciones que podrían convertirse en un futuro en lecciones o premisas para campañas online dentro del marco de la Unión Europea.

\subsection{Efectos secundarios del Brexit y city branding}

El 23 de junio de 2016, Reino Unido celebró un controvertido referéndum en el que una estrecha mayoría del electorado optó por abandonar la Unión Europea tras cuatro décadas de pertenencia. Desde entonces, existe un grado de incertidumbre considerable con respecto al proceso del acuerdo de retirada (Llaudes et al., 2018; Tuñón-Navarro; Carral, 2018). Si bien no podemos cuantificar todavía todas las consecuencias del Brexit, diferentes académicos han apuntado:

- incertidumbre,

- recesión económica tanto para la Unión Europea como para el Reino Unido,

- un enfoque de cooperación más que de pertenencia,

- barreras comerciales,

- posible acceso al mercado interior a cambio del mantenimiento de las libertades de la UE en el Reino Unido,

- un posible efecto de contagio, y

- una mayor cohesión de la Unión Europea y equilibrio de poder (Bouza-García, 2017; Patel; Reh, 2016; Tuñón-Navarro; Carral, 2018; Tuñón-Navarro; Bouza-García; Carral, 2019).

No obstante, otros asuntos (efectos secundarios) tenían que resolverse en la primera fase de negociaciones, incluyendo decisiones sobre la reubicación de agencias de la Unión Europea hasta ahora establecidas en Londres, tales como la Agencia Europea de Medicamentos (EMA), que se trasladó a Amsterdam, y la Autoridad Bancaria Europea (EBA) a París. Dadas sus dimensiones y trascendencia, albergar la EMA era considerada la joya de la corona de los efectos secundarios del Brexit: una joya para cualquiera de los 19 candidatos originales para suceder a Londres, asumiendo una plantilla de 900 personas con sus respectivas familias, un presupuesto de alrededor de 320 millones de euros al año y la tarea de evaluar y autorizar la comercialización de nuevas medicinas en la UE. Dada esta oportunidad, y a través de un particular proceso de selección, 19 ciudades europeas solicitaron convertirse en la nueva sede de la EMA. Durante un proceso que duró casi cinco meses (del 22 de junio hasta el 20 de noviembre de 2017), los candidatos llevaron a cabo campañas políticas y de marketing para demostrar su convergencia con los criterios de selección oficiales. Con más o menos apoyo 
de sus países respectivos, las ciudades candidatas empezaron una intensa campaña de comunicación institucional para captar el mayor número de votos.

Entre las múltiples herramientas de marketing o branding organizacional a escala municipal descritas en la bibliografía (Lara-Pacheco, 2015; Fernández-Falero et al., 2017, Campillo-Alhama; Martínez-Sala, 2017), este estudio se enfoca en el branding online a través de los portales web ad hoc de los candidatos. Hunde por tanto también sus raíces en la propia literatura del city branding o la mercadotecnia de ciudades. De hecho, las ciudades llevan a cabo estrategias políticas, pero también de comunicación para tratar de dotarse de una identidad propia para obtener reconocimiento internacional (Zapata, 2007, p. 81), así como para competir y atraer inversiones para el territorio (Van-der-Pluijm; Melissen, 2007, p. 13; Lara-Pacheco, 2015, p. 226). Particularmente, dicha competencia y apertura a innovaciones e inversiones ha provocado que las áreas metropolitanas desarrollen estrategias de atractividad territorial, ancladas, al menos, en un triple eje:

- desarrollo económico: ofreciendo condiciones de recepción de inversiones lo suficientemente atractivas para la ubicación de los proyectos con preferencia a otras jurisdicciones (Hatem, 2004, p. 1);

- paradiplomacia o proyección de la estrategia de comunicación de la marca territorio (Lara-Pacheco, 2015, pp. 227-228);

- perspectiva cultural y narrativa: que supone la capacidad de segmentar audiencias, movilizando recursos culturales adecuados que realcen las ventajas comparativas y los imaginarios narrativos que tienen dichos relatos para las audiencias a los que se dirigen (Cusin; Damon; 2012, p. 89; Amendola, 2000, p. 287).

Es por ello que las ciudades también desarrollan planes y estrategias de comunicación organizacional, denominadas incluso como de place branding (Kotler; Armstrong, 2001, p. 249; Kavaratzis; Asworth, 2010). Pretenden así, entre otros objetivos, la recepción de inversiones y la reputación en calidad de áreas de negocios, en una estrategia de comunicación organizacional de posicionamiento, destinada tanto al interior como al exterior del territorio (Ward, 2005, p. 1).

\subsection{Comunicación institucional en el contexto europeo}

Los últimos incidentes o "policrisis" (euro, refugiados y Brexit) alterando la evolución de la UE han puesto en manifiesto también el fracaso de la política de comunicación de la organización (Papagianneas, 2017; Tuñón-Navarro, 2017; Tuñón-Navarro; Bouza-García; Carral, 2019). A escala de comunicación organizacional Europa está ante una encrucijada que le ofrece una oportunidad única para renovar el mensaje, homogeneizarlo y segmentarlo en función de las características contemporáneas de las audiencias a las que se dirige.

Por ejemplo, es esencial para la política de comunicación de la UE modificar sus normas para proveer acceso a las metodologías tecnológicas emergentes, especialmente online y a través de las redes sociales. Mientras varios académicos han analizado el discurso político sobre los nuevos medios y las redes sociales como Twitter (Eom et al., 2015; Kreiss, 2016, Larsson; Kalsnes, 2014), tratamos de transferir este análisis al campo transnacional europeo online (basado en webs). De hecho, junto con la información proveniente de las mismas instituciones comunitarias, los países miembros comparten la tarea de comunicar. Por consiguiente, las fórmulas online son ahora cruciales para los actores políticos e institucionales para comprometer a los ciudadanos e involucrar a las audiencias (Campos-Domínguez, 2017; López-Meri; Marcos-García; Casero-Ripollés, 2017) como herramientas esenciales de estrategia de comunicación política de la UE (Papagianneas, 2017), o de los dirigentes europeos (Bouza-García; Tuñón-Navarro, 2018).

Asimismo, tiene recorrido en la comunicación política trasnacional europea online la personalización, faceta que ha recibido ya una notable atención académica y que supone una aproximación del discurso enfocada en las personas, anteponiendo las cualidades intrínsecas de los oradores sobre el contenido o las ideologías de sus discursos, es decir la forma sobre el contenido (Sánchez-Murillo, 2005; López-Meri; Marcos-García; Casero-Ripollés, 2017), incluso específicamente en redes sociales (López-García, 2016), habiendo atendido recientemente algunas a la comunicación institucional (europea) del propio presidente francés, Enmanuel Macron (Habermas, 2017; Bouza-García; Tuñón-Navarro, 2018). Además, dentro del propio marco transnacional europeo, cada vez son más las investigaciones que inciden sobre campañas online, bien desde la perspectiva del supuesto déficit democrático (Davesa; Shahin, 2014), de los movimientos sociales (Bouza-García; Oleart; Tuñón-Navarro, 2019), o de las consultas ciudadanas (Quittkat, 2011).

\subsection{Comunicación organizacional y narrativas digitales}

La comunicación institucional de los gobiernos está en la actualidad en una encrucijada de diferentes enfoques metodológicos. Aunque existen relativamente escasos estudios en el campo de las relaciones públicas y la comunicación organizacional (especialmente vinculada a la comunicación institucional), algunos de sus enfoques conceptuales pueden aplicarse a la investigación de comunicación gubernamental. En particular, el branding, la reputación y la comunicación simétrica pueden constituir métodos esenciales para la profundización en la comunicación institucional gubernamental (Canel; Sanders, 2012).

Particularmente, la narrativa se ha convertido en un elemento clave de la comunicación organizacional online durante los últimos años (Maarek, 2014; D’Adamo; García-Beaudox, 2016; o García-Orosa; Vázquez-Sande; López-García, 2017). Asimismo, el desarrollo de las nuevas tecnologías de la información y la comunicación e internet (Serrano-Cobos, 2016), además de la innovación y la experimentación (Salaverría, 2015; Peñafiel, 2016), han vinculado definitivamente la comunicación organizacional a las narrativas digitales, evolucionando desde unas primeras eminentemente textuales has- 
ta las más recientes, conceptualizadas como multiplataforma (Guerrero-Pico; Scolari, 2016).

En el marco de un contexto de cambio y de nueva comprensión de la narrativa en sentido expansivo, se ha venido construyendo un corpus de trabajos académicos que han pretendido precisamente el análisis de esas nuevas narrativas, dentro de los diferentes subsectores de la comunicación corporativa:

- partidos políticos (Maarek, 2014; Casero-Ripollés; Feenstra; Tormey, 2016; Larrondo-Ureta, 2016; López-García, 2016; o García- Orosa; Vázquez-Sande; López-García, 2017; entre otros);

- comunicación política (Scolari et al., 2012);

- ámbito municipal (Fernández-Falero et al., 2017, Campillo-Alhama; Martínez-Sala, 2017; López-Alonso; Moreno-López, 2019);

- marco de las ONGs (Marfil-Carmona, 2013; Martínez-Valero, 2016; Arroyo-Almaraz; Calle-Mendoza; Van-Wyk, 2018);

- ámbito empresarial (Costa-Sánchez, 2014).

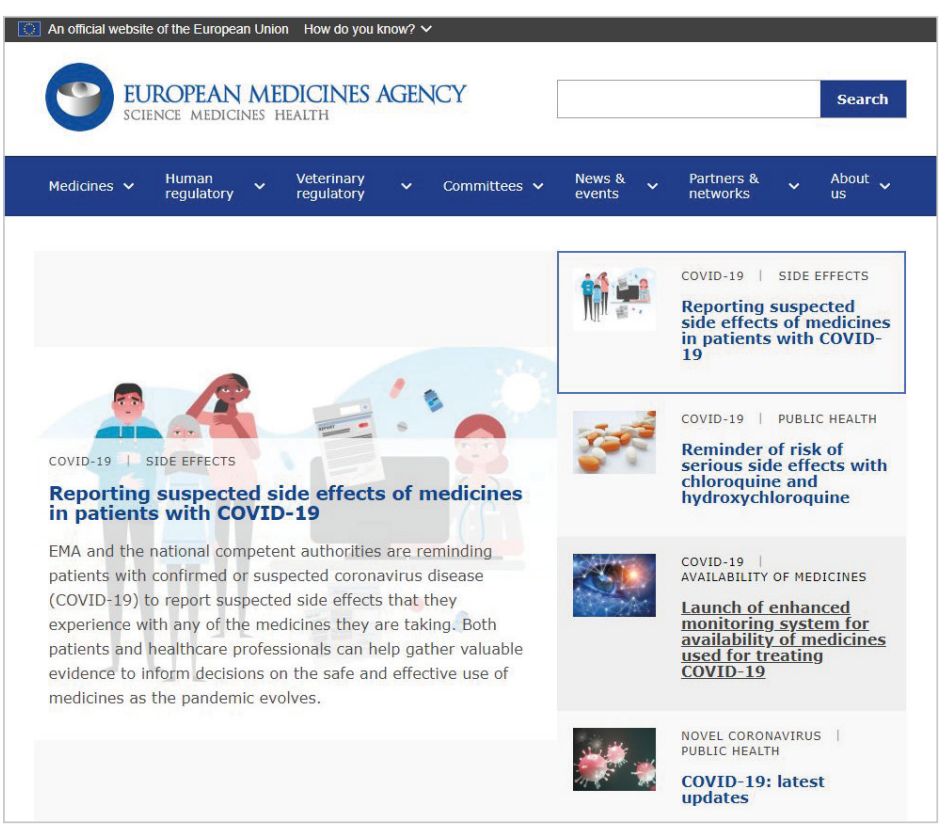

https://www.ema.europa.eu

Como ha podido comprobarse, el campo de los asuntos europeos no ha constituido hasta la fecha un área tradicional de investigación en las narrativas y la comunicación organizacional, motivo por el que esperamos poder contribuir a cerrar este gap desde un enfoque metodológico multidisciplinar.

Apoyándose en las innovaciones derivadas de los cambios tecnológicos se han acuñado términos definitorios del uso de las nuevas herramientas tecnológicas en la comunicación política, siendo de los más celebrados el de la tecnopolítica (Gutiérrez-Rubí, 2014), que incide en la necesidad de nuevos lenguajes y narrativas. De hecho, profundizar en estos usos tecnopolíticos y evaluar su eficacia representa una prioridad, tanto a nivel profesional, como académico (Gutiérrez-Rubí, 2014). Los departamentos de comunicación (principalmente de los partidos políticos) asumen hoy la necesidad de orquestar múltiples acciones y de hacerlas converger en la búsqueda de un mismo resultado (Larrondo-Ureta, 2016). De hecho, en estos departamentos una clara evolución marcada por estrategias específicas para el medio con mayor poder de proyección, la web (García-Orosa, 2009; Túñez-López, 2012), que progresivamente viene siendo acompañada o/y reemplazada por las redes sociales, es lo que obliga a los departamentos de comunicación a afrontar el desafío de comenzar a crear campañas de comunicación organizacional online omnicomprensivas.

\section{Metodología, base de datos y preguntas de investigación}

Este artículo se basa en un análisis de contenido de las narrativas organizacionales de las ciudades candidatas a través de sitios online ad hoc durante el proceso de selección de reubicación de la EMA. Nos enfocamos en 16 casos de estudio (Amsterdam, Atenas, Barcelona, Bonn, Bratislava, Bruselas, Bucarest, Copenhague, Helsinki, Lille, Malta, Milán, Oporto, Estocolmo, Viena y Zagreb) de los 19 candidatos, ya que tres de ellos -Sofía, Varsovia y Dublín- no proporcionaron un sitio online ad hoc. Se eligió el análisis de contenido como el planteamiento metodológico para este estudio ya que facilita la codificación y la cuantificación sistemática de los mensajes institucionales de los candidatos y permite la identificación de tendencias. El análisis de contenido ha sido usado frecuentemente como un método descriptivo para identificar las características de los mensajes (Iyengar; Simon, 2000). Más allá de la descripción, también resulta útil para la interpretación y la generalización de patrones.

\section{Esta investigación compara y contrasta campañas políticas/organizacionales on- line y destaca los marcos y variables en cuestión durante el proceso de selección de la EMA}

El análisis de contenido de los anteriores materiales de comunicación organizacional se realizó por medio de NVivo (v.12 plus). Se elaboró un esquema de codificación personalizado y un conjunto de reglas de codificación. El sistema de codificación se diseñó para identificar las frecuencias de los temas que se consideran puntos álgidos en política, tal como:

- la accesibilidad y la ubicación,

- negocios y oportunidades de inversión,

- comunidades de expatriados,

- el sector sanitario y científico,

- la calidad de vida, prensa y marketing,

- capacidades para la transición de la reubicación,

- presentación del lugar, entre otros. 
Nuestro análisis de contenido determinó la frecuencia de estos temas, así como sus asociaciones con otros asuntos o mensajes similares que pueden referirse a más de un tema. Ello se consiguió usando nodos de matrices. Una matriz de nodos tabula de manera cruzada el número de contenidos codificados (o nodos) a través de categorías y capta la correlación entre términos clave.

Sólo fue codificado el material relacionado directamente con la reubicación de la EMA, excluyéndose otros temas colaterales que pudieran haber tenido un impacto indirecto, o aquellos incluidos en folletos o catálogos hipervinculados a los sitios web, pero no incluidos dentro de los mismos.

Diseñamos tres fases de investigación (Bardin, 2002):

- Pre-análisis: implicó la implementación de temas y categorías para la codificación. Este procedimiento se realizó de manera ad hoc: era imposible simplemente transferir los modelos o categorías de otros estudios acerca de la comunicación institucional de los gobiernos, debido a la excepcionalidad y la especificidad del sistema de reubicación de la EMA (Unión Europea, 2017). Primero se definieron los temas e indicadores que fueron derivados de forma deductiva de conceptos teóricos y trabajos publicados previamente, para después complementarse, de manera inductiva, a través de una lectura superficial del contenido de webs. Una vez que se estableció la serie provisional de temas coincidentes en las estrategias organizacionales de las candidaturas, la misma fue examinada, discutida y modificada por el equipo de investigación. La fase de pre-análisis incluyó un test piloto, una consulta con expertos y una metodología de consenso. Tres expertos en comunicación evaluaron la versión revisada. Los expertos dieron a los elementos de estudio valores de 4 ó 5 en una escala Likert de 1 a 5 . Finalmente, los co-autores determinaron el esquema de codificación final basándose en las reacciones de los expertos.

- Examen del material: consistió en la lectura pormenorizada del contenido textual de las webs y la codificación de cada uno de los casos de estudio. Una vez que los co-autores cumplimentaron la codificación de los materiales comunicativos, se llevó a cabo una reunión para llegar a un consenso dondequiera que hubiera discrepancias. Cuando no hubo consenso, se consultó a un tercer investigador (un experto en comunicación institucional gubernamental), hasta alcanzarse una completa conformidad del $100 \%$.

- Análisis e interpretación de resultados: se utilizó un enfoque cuantitativo con la ayuda del paquete estadístico SPSS 20, que se complementó con el análisis de discurso apoyado en la utilización del software NVivo v.12.

Por último, se debería destacar que el proceso de reubicación de la EMA (Unión Europea, 2017) se decidió a través de un sistema de votaciones por el que los países concedían uno, dos o tres puntos a otros candidatos en diferentes rondas. Desde un punto de vista metodológico, consideramos candidatos exitosos (en comparación con el resto de candidatos) a aquellos que pasaron a la segunda ronda (Amsterdam, Milán y Copenhague). Por lo tanto, debe señalarse que, en la primera ronda, Milán recibió un total de 25 puntos mientras que Amsterdam y Copenhague recibieron 20 votos cada una. Bratislava recibió 15; Barcelona 13; Estocolmo 12, Atenas 10; Oporto 10; Varsovia y Bucarest 7; Bruselas y Helsinki 5; Viena 4 y Bonn; Lille y Sofía 3. En la segunda ronda, se eliminó a Copenhague. Ni Milán ni Amsterdam pudieron conseguir una mayoría. Finalmente, Amsterdam obtuvo la EMA tras lanzar una moneda al aire, al terminar con el mismo número de votos que Milán, en la tercera ronda de la votación.

Los datos se extrajeron de las webs oficiales primarias de las 16 ciudades aspirantes a la reubicación de la EMA durante las dos semanas previas a la elección de Amsterdam.

http://www.netherlandsforema.eu
http://emathens.eu
http://bcn4ema.eu/index.html
http://www.closer-to-europe.eu/en-10899\#106342
http://www.sk4ema.sk
http://www.emabe.eu
http://www.emabucharest.ro
http://emacph.eu
http://emahelsinki.fi
http://www.emainlille.eu/en
http://ema.mt
http://www.emamilano.eu
https://emainporto.eu
https://www.ematosweden.eu
https://viennabusinessagency.at/international/emazing-vienna/ema-in-vienna
http://www.emainzagreb.eu

En comparación con las plataformas de redes sociales, el análisis de sitios web permitió un acceso mucho más comprensivo y datos homogéneos. De hecho, las webs de las ciudades candidatas usaron una plantilla similar de Wordpress, fa- 
cilitando la extracción y recopilación de datos. Los datos recogidos incluyeron textos, fotos, vídeos, mapas, logos, infografías y/o catálogos de cada uno de los 16 candidatos mencionados anteriormente.

La codificación de los indicadores elaborados durante la toma de muestras abordaron las siguientes variables de una naturaleza cualitativa y cuantitativa, mediante las cuales se identificaron estrategias de comunicación organizacional/institucional online a nivel técnico y en términos de comunicación política gubernamental:

- actores involucrados (interesados);

- áreas temáticas predominantes (contenido y encuadre);

- receptores de mensajes (audiencias);

- cumplimiento de los criterios de selección (oferta técnica);

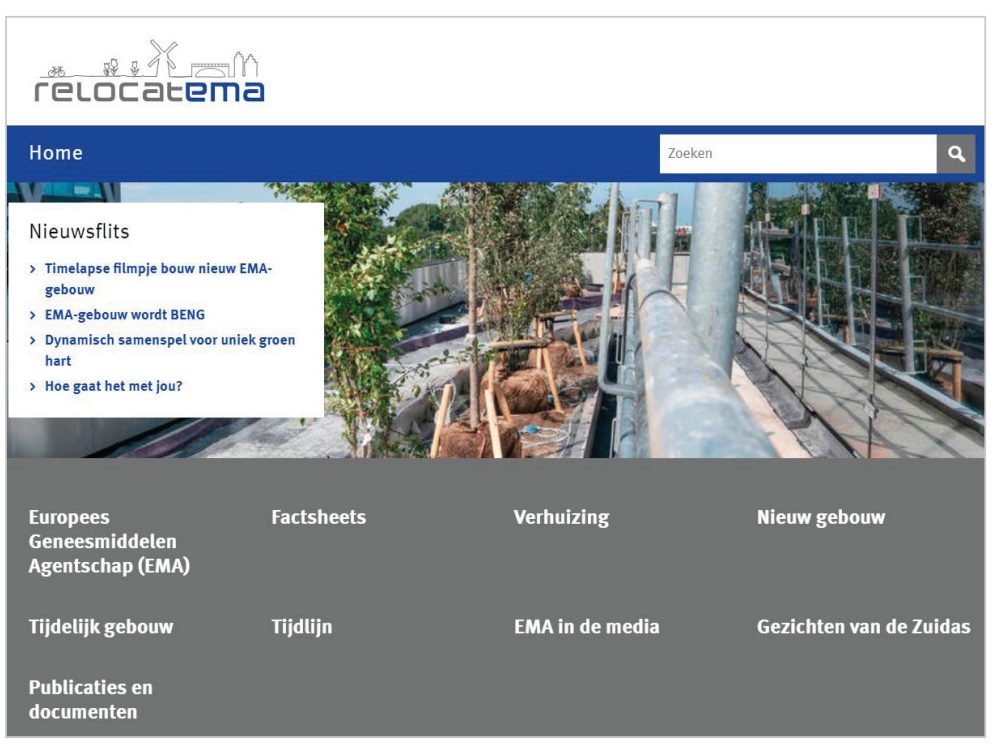

Los materiales de la candidatura de Amsterdam a albergar la EMA, se conservan en este archivo: $h$ ttps://relocatema.archiefweb.eu

- estructura de la web (organización del sitio online);

- otros materiales tales como vídeos, audios, fotos, mapas e infografías (dimensión hipertextual);

- comentario y contacto (retroalimentación con las audiencias);

- lenguas (participación del público);

- enlaces a otras webs y/o redes sociales (engagement).

El conjunto total de datos comprendió un texto escrito de aproximadamente $\mathrm{N}=62.129$ palabras, repartidas de forma desigual entre las ciudades candidatas: la tabla 1 muestra esta diferencia cuantitativa entre las campañas de estrategia, desde la más extensa (Lille: 15.426 palabras) a la más reducida (Copenhague: 151 palabras). La extensión media fue de cuatro mil palabras por candidata (concretamente 3.916), cifra utilizada como referencia para definir las estrategias como más o menos extensas, en cuanto a su dimensión textual.

Tabla 1. El texto de las webs de los candidatos de la EMA (palabras) y video (segundos) longitud, y fotografías y otros recursos visuales usados

\begin{tabular}{|l|c|c|c|c|}
\hline \multicolumn{1}{|c|}{ Ciudad candidata } & $\begin{array}{c}\text { Extensión texto } \\
\text { (palabras) }\end{array}$ & $\begin{array}{c}\text { Duración videos } \\
\text { (segundos) }\end{array}$ & Fotos & Otros recursos visuales \\
\hline Amsterdam & 5.397 & 295 & 36 & 17 \\
\hline Atenas & 6.381 & 0 & 6 & 1 \\
\hline Barcelona & 545 & 311 & 24 & 3 \\
\hline Bonn & 1.705 & 192 & 42 & 3 \\
\hline Bratislava & 7.841 & 127 & 25 & 8 \\
\hline Bruselas & 4.000 & 0 & 31 & 3 \\
\hline Bucarest & 3.696 & 270 & 1 & 5 \\
\hline Copenhague & 151 & 172 & 4 & 5 \\
\hline Helsinki & 1.197 & 116 & 13 & 5 \\
\hline Lille & 15.426 & 144 & 32 & 8 \\
\hline Malta & 2.988 & 324 & 27 & 6 \\
\hline Milán & 4.848 & 158 & 28 & 1 \\
\hline Oporto & 1.774 & 190 & 17 & 0 \\
\hline Estocolmo & 2.988 & 544 & 43 & 6 \\
\hline Viena & 1.018 & 0 & 478 & 0 \\
\hline Zagreb & 2.714 & 262 & & 79 \\
\hline Total & 62.669 & $51,95 m i n$ & & \\
\hline
\end{tabular}

Además, el conjunto de datos comprendió 24 vídeos, alcanzando un total de 51 minutos y 45 segundos (3.105 segundos). La tabla 1 destaca cómo Estocolmo subió cinco vídeos con una duración total de 9,04 minutos, mientras que otros candidatos (Viena, Varsovia, Sofía, Dublín, Bruselas y Atenas) no exhibieron ningún vídeo.

Asimismo, nuestros datos comprendieron 478 fotografías. Como evidencia la tabla 1, su uso fue variado. Mientras que Copenhague sólo ilustró su estrategia organizacional con una foto, la candidata francesa Lille atestó su web con 104 imá- 
genes. Algunas estrategias online incluyeron otros recursos visuales menos comunes, tales como mapas, infografías y tablas, totalizando 79, que fueron usados irregularmente. La tabla 1 muestra que muchas de las ciudades candidatas no las usaron, y la ganadora final, Amsterdam (17), al igual que Atenas, Bruselas y Malta (8 cada una) fueron las que más.

Todos los datos online (textos, fotos, vídeos, mapas, infografías y tablas) se codificaron manualmente, verificándose variables capitales tales como:

- temas y encuadres,

- actores predominantes,

- audiencias,

- declaración de conformidad con los criterios seleccionados.

Se llevaron a cabo búsquedas de texto y un análisis de frecuencia de palabras suplementario asistido por ordenador (mediante NVivo 12 plus) para medir las frecuencias temáticas, así como un análisis de contenido adicional. La tabla 2 muestra las 20 palabras más frecuentes en los textos analizados (excepto los de Copenhague cuyo número no era suficiente para indicar 20 palabras frecuentes). La primera fila muestra la frecuencia de los llamados candidatos menos exitosos (los 13 que no pasaron la primera ronda), mientras que la segunda indica la frecuencia entre los candidatos exitosos (Amsterdam, Milán y Copenhague). La tercera, cuarta y quinta se dedican específicamente a las ciudades candidatas más exitosas.

Tabla 2. Análisis de las palabras más frecuentes de las webs de los candidatos a la EMA (NVivo codificación):

*Sólo se seleccionaron nombres. Verbos, pronombres, preposiciones y adverbios fueron excluidos;

**Se incluyeron plurales y/o sinónimos.

Palabras* más frecuentes en los textos online de las ciudades candidatas menos exitosas

Palabras* más frecuentes en los textos online de las ciudades candidatas más exitosas

Palabras* más frecuentes en los textos online de las ciudades candidatas más exitosas (Amsterdam)

Palabras* más frecuentes en los textos online de las ciudades candidatas más exitosas (Milán)

Palabras* más frecuentes en los textos online de las ciudades candidatas más exitosas (Copenhague)

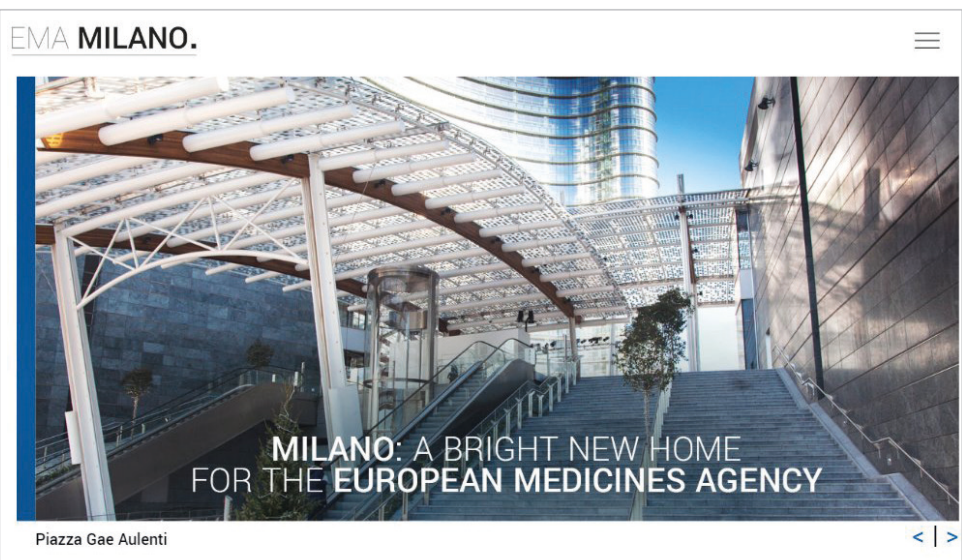

With one of Europe's best research and business environment, excellent links with Europe and the World, a vibrant world-class lifestyle as well as superb conditions for an efficient relocation, the candidacy of Milan fully supports the objective of ensuring continuity in the activity of the European Medicines Agency (EMA) during the post-Brexit transition from London

WHY MILANO / MILANO FOR EMA

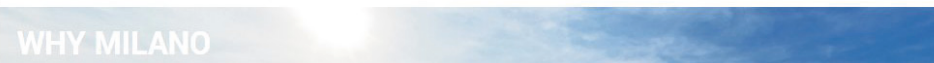

Web de la candidatura de Milán a albergar la EMA, aún accesible en abril de 2020. http://www.emamilano.eu

Este análisis muestra algunas palabras clave que pueden representar las estrategias online de contenido de los candidatos. De hecho, se ha encontrado un tipo de patrón común entre las ciudades candidatas más y menos exitosas, ya que coinciden en un 65\% de los casos (EMA, europeo, ciudad, internacional, ofrece, agencia, mundo, salud, área, nuevo, escuela, Europa y ubicación). Por consiguiente, las palabras clave más frecuentes no son exclusivas ni para las candidatas más exitosas.

Respecto al contenido, cabe también destacar que las palabras clave estaban más relacionadas con asuntos políticos/ administrativos generales que científicos o de salud. De hecho, las palabras más frecuentes relacionadas con la salud eran pocas (investigación, medicinas y salud para las menos exitosas, y sólo salud para las exitosas). Este acercamiento también se puede verificar en el análisis de cada caso de las candidatas más exitosas (Amsterdam, Milán y Copenhague), donde también se contrastó que las palabras más frecuentes estaban relacionadas con los propios países de origen.

En definitiva, a través de un análisis de contenido de los sitios online oficiales de los candidatos, que incide preferentemen- 
te sus dimensiones cuantitativas, este artículo examina las estrategias online de las ciudades candidatas. El análisis trata de resolver dos temas clave:

- esclarecer cómo se diseñaron estas estrategias online;

- descubrir si había una tendencia, lógica o relación clara entre las estrategias online elaboradas con más recursos y frecuencias (teorías resource push sobre resource pull) (Jones; Keating, 1995; Rhodes, 1995; Tuñón-Navarro, 2009) y el éxito del concurso final.

Se busca validar así la siguiente pregunta de investigación o hipótesis:

- Ha habido un patrón común entre las estrategias de comunicación organizacional online de los candidatos más exitosos en el proceso de reubicación de la EMA. Por ello, las candidatas más exitosas exhibieron una estrategia online más prolífica que sus rivales.

Además, la pregunta de investigación puede desagregarse entre cuatro sub-hipótesis directamente relacionadas con:

- actores involucrados;

- audiencias a las que el mensaje se dirige;

- mensajes o temas predominantes;

- criterios requeridos presentados en las estrategias online de los candidatos.

Por lo tanto:

- Actores: la variedad, el número y el perfil político de los actores representados en las estrategias online favorecieron el éxito del candidato;

- Audiencias: los candidatos más exitosos fueron aquellos que dirigieron el mensaje preferentemente a la Unión Europea y los empleados de la EMA;

- Temas: es posible verificar una lógica o relación entre los temas preferentes usados por los candidatos más exitosos en función de la variedad y/o la definición de los mismos;

- Criterios: tanto los candidatos más como los menos exitosos cumplieron los criterios requeridos de una manera homogénea en sus estrategias online, sin ser esto un elemento diferenciador.

\section{Resultados y discusión}

Aparte de la cuantificación (tabla 1), nuestro análisis tuvo dos niveles:

- codificamos la información correspondiente a contenido puro, desde una doble perspectiva: la más específica verificación de declaración de conformidad con los criterios, y la más genérica disección de los temas principales o encuadres más generales verificados;

- el segundo nivel de análisis no destacó el "qué", sino el "quién”, para lo que incidió tanto en los actores involucrados en las estrategias online, por un lado, como en las audiencias seleccionadas de las estrategias online, por el otro.

\subsection{Temas preferentes y cumplimiento de los criterios}

Buscamos encuadrar y codificar nuestros textos en función de los temas principales, los cuales identificamos como:

- presentación del lugar;

- accesibilidad y ubicación;

- sector sanitario;

- calidad de vida en la ciudad candidata;

- comunidades expatriadas;

- prensa y marketing;

capacidades de transición en la reubicación;

- negocios y oportunidades de inversión.

Este enfoque de encuadre indicó que:

- "la calidad de vida en la nueva ciudad" era el tema más frecuente y atractivo entre las ciudades candidatas (indicado por 15 de las 16 webs, o el 93,75\%);

- el "sector sanitario" y la "presentación del lugar" ocuparon el segundo puesto (11 de las 16 webs, o el 68,75\%);

- "accesibilidad y ubicación" y "capacidades de transición de la reubicación” ocupan el tercer puesto (43,75\%), seguido de

- "comunidades expatriadas" y "negocio y oportunidades de inversión" (31,25\%), y finalmente

- "prensa y marketing" (18,75\%).

Aunque algunas estrategias de campaña presentaron varias categorías transversales, destacamos los ejemplos más evidentes. Estas categorías se desarrollaron usando un planteamiento inductivo, conducido por las preguntas de investigación. Además, atendiendo a las nociones de la estrategia de encuadre político (Aalberg; Strömbäck; De-Vreese, 2012), 
codificamos nuestros datos en función de su presentación positiva o negativa de argumentos temáticos de peso o de la ausencia de los mismos.

Tabla 3. Principales temas de las webs de los candidatos a la EMA

\begin{tabular}{|c|c|c|c|c|c|c|c|c|c|c|c|c|c|c|c|c|}
\hline & AMS & ATE & $\mathrm{BCN}$ & BON & BRA & BRU & BUC & COP & HEL & LIL & MAL & MIL & OPO & EST & VIE & ZAG \\
\hline Presentación de la sede & $x$ & $x$ & & & $x$ & $x$ & $x$ & & $x$ & & & $x$ & $x$ & & & $x$ \\
\hline $\begin{array}{l}\text { Accesibilidad y locali- } \\
\text { zación }\end{array}$ & $x$ & & $x$ & $x$ & & & $x$ & $\mathbf{x}$ & & $x$ & & $x$ & & $x$ & $x$ & \\
\hline Sector sanitario & $\mathbf{x}$ & $x$ & & $x$ & & $x$ & $x$ & $\mathbf{x}$ & $x$ & $x$ & & $x$ & & $x$ & & $x$ \\
\hline Calidad de vida & $\mathbf{x}$ & $x$ & & $x$ & & $x$ & $x$ & & $x$ & $x$ & $x$ & $\mathbf{x}$ & $x$ & $x$ & & $x$ \\
\hline $\begin{array}{l}\text { Comunidades de expa- } \\
\text { triados }\end{array}$ & $x$ & & $x$ & & & & & $x$ & & & & $x$ & & & $x$ & $x$ \\
\hline Marketing y prensa & & & & $x$ & & & $x$ & & & $x$ & & & & & & \\
\hline $\begin{array}{l}\text { Capacidades de transi- } \\
\text { ción de la reubicación }\end{array}$ & $x$ & & $x$ & $x$ & & $x$ & & & $x$ & & & & & & & $x$ \\
\hline $\begin{array}{l}\text { Opciones de negocio e } \\
\text { inversión }\end{array}$ & & & $x$ & & & & & $x$ & & & & $X$ & & & $X$ & \\
\hline Otros & & & & & $x$ & & & & & & $x$ & & & & & $x$ \\
\hline
\end{tabular}

La tabla 3 revela información valiosa sobre la estrategia de selección temática de contenidos de cada candidato. De hecho, sus estrategias online difirieron en términos de extensión y prioridades. Se ha verificado un tipo de tendencia entre aquellas estrategias que encuadran más temas y su éxito final. Precisamente, Amsterdam y Milán encuadraron hasta seis categorías (el promedio total fue de cuatro). Sin embargo, Copenhague (otro candidato que pasó la primera ronda) sólo encuadró cuatro temas (promedio), y Zagreb (un candidato no exitoso) diseccionó hasta seis, como Amsterdam y Milán.

Por otra parte, para confirmar los resultados anteriores, optamos por verificar la fiabilidad a través de un intercoder. Los resultados indican la frecuencia promedio de los encuadres en el texto online. Se verificaron diversas estrategias entre las candidatas exitosas: fueron más extensas las de Amsterdam y Milán, y más restringida en el caso de Copenhague. Milán y Amsterdam asignaron la preferencia más alta a la calidad de vida, seguido de comunidades expatriadas, accesibilidad y ubicación. Sin embargo, los sectores de salud y negocios fueron prioritarios para Milán, mientras que Amsterdam intensificó su interés por las capacidades para la transición en la reubicación. Copenhague no priorizó la calidad de vida sino las comunidades expatriadas, seguidas del sector sanitario, accesibilidad y negocios.

Tabla 4. Principales temas de las webs de los candidatos a la EMA más exitosos

\begin{tabular}{|l|c|c|c|}
\hline \multicolumn{1}{|c|}{ Temas } & Amsterdam & Milán \\
\cline { 2 - 3 } & & \multicolumn{2}{c|}{ Copenhague } \\
\hline Presentación de la sede & 3,92 & 10,78 \\
\hline Accesibilidad y localización & 19,48 & 17,31 \\
\hline Sector sanitario & 8,27 & 18,57 \\
\hline Calidad de vida & 29,67 & 26,76 \\
\hline Comunidades de expatriados & 25,65 & 17,44 \\
\hline Marketing y prensa & - & - \\
\hline Capacidades de transición de la reubicación & 24,06 & - \\
\hline Opciones de negocio e inversión & - & $-23,22$ \\
\hline Otros & - & 10,26 \\
\hline
\end{tabular}

Los datos también destacaron cómo las estrategias online de los candidatos intentaron demostrar su cumplimiento de los criterios de selección de la UE. Aquí se pueden destacar varios resultados muy homogéneos. Se verificó cada criterio para una gran mayoría de los candidatos. 13 de los 15 candidatos demostraron su cumplimiento con los criterios del 1 al 5:

- disponibilidad de la sede desde la consumación del Brexit;

- accesibilidad a la localización;

- facilidades educativas para los hijos de los empleados de la agencia;

- acceso al mercado de trabajo, seguridad social y atención médica para los familiares de los empleados; y

- aseguramiento de la continuidad del trabajo de la agencia-. 
El sexto criterio (distribución geográfica) fue defendido sólo por 3 de las ciudades candidatas. En efecto, como fue verificado a través de NVivo de forma adicional, con la excepción del 6으 criterio, los sitios web de los candidatos cubrieron los criterios requeridos de una manera homogénea, sin ser esto un elemento diferenciador, independientemente de haber mostrado una estrategia más o menos extensa o de haber resultado más o menos exitosas.

\subsection{Actores y audiencias}

El segundo nivel de análisis destacó las estrategias de los actores y sus audiencias seleccionadas. De este modo, los actores comprometidos en las estrategias de campaña online fueron analizados cuidadosamente, no sólo cuantitativa, sino también cualitativamente (políticos, funcionarios públicos, académicos y hombres de negocio, entre otros). Se ha comprobado que los políticos fueron los actores más visibles: ya que hasta un $77 \%$ de las estrategias online involucraron a políticos y/o funcionarios públicos, seguidos de académicos y/o investigadores (15\%), y finalmente por hombres de negocios (8\%). Debe señalarse que periodistas, sindicatos y asociaciones no estuvieron involucrados en la campaña online oficial.

Además, identificamos claras divergencias con respecto a la variable actores, ya que la mitad de los candidatos optaron por introducirlos para apoyar sus estrategias online (Amsterdam, Bonn, Bratislava, Bucarest, Copenhague, Lille y Estocolmo), mientras que la otra mitad no utilizó dicha opción. Copenhague y Bratislava no incluyeron estos actores en su estrategia de texto online, pero sí en vídeos y catálogos. Por consiguiente, permanecieron

El acercamiento de encuadre reveló que la calidad de vida en una ciudad nueva fue el tema más frecuente y atractivo entre las ciudades candidatas, mientras que el sector sanitario y la presentación del lugar constituyeron los segundos temas

fuera de la evaluación de esta variable. Entre los candidatos que introdujeron actores en sus estrategias online, nuestro análisis de NVivo encontró algunas tendencias destacables.

De los seis candidatos que dieron voz a los diferentes actores mediante sus estrategias de texto online, sólo Amsterdam pasó la primera ronda. Por consiguiente, no se puede deducir que los candidatos más exitosos introdujesen más actores, ya que Milán y Copenhague tenían una estrategia muy limitada en estos términos.

El análisis asistido por ordenador detectó la frecuencia con la que los candidatos introdujeron actores en sus estrategias online. Una estrategia extensa podría verificarse en los casos de Bonn (19,86\%), Bucarest (22,76\%) y Lille (29,61\%); y una más restringida para Amsterdam (4,39\%), Atenas (8,25\%) y Estocolmo (6,02\%), según puede advertirse en la tabla 5.

Por un lado, el software informático sirvió como intercoder para verificar la prominencia de los políticos y funcionarios públicos como actores en cada caso, con la excepción de Lille, cuya candidatura fue principalmente apoyada por investigadores y académicos. De hecho, los investigadores y académicos constituyeron la segunda categoría más frecuente de actores, aunque su impacto se limitó a Amsterdam, Bucarest y Lille; mientras que los hombres de negocios fueron los únicos usados como actores por Lille.

Por otro lado, NVivo 12 Plus se usó también para verificar (caso por caso) la aparición de actores en las estrategias de comunicación organizacional:

- Lille usó todas las categorías de actores;

- Bonn y Bucarest optaron por estrategias extensas, pero sin satisfacer todas las categorías de actores;

- Atenas, Bonn y Estocolmo sólo introdujeron una categoría (políticos y funcionarios públicos);

- Amsterdam introdujo dos categorías, pero dentro de una estrategia general muy restringida.

Por consiguiente, no se verificó si las estrategias más exitosas introdujeron actores más a menudo en términos de cantidad o variedad, que las candidatas menos exitosas.

Tabla 5. Frecuencia promedio de aparición de actores en las webs de la EMA por tipo

\begin{tabular}{|c|c|c|c|c|c|c|}
\hline \multirow{2}{*}{ Tipo de actor } & Amsterdam & Atenas & Bonn & Bucarest & Lille & Estocolmo \\
\hline & \multicolumn{6}{|c|}{$\%$} \\
\hline Políticos / servidores públicos & 3,13 & 8,25 & 19,86 & 18,63 & 3,90 & 6,02 \\
\hline Académicos / investigadores & 1,26 & - & - & 4,16 & 16,93 & - \\
\hline Hombres de negocios & - & - & - & - & 8,78 & - \\
\hline Total & 4,39 & 8,25 & 19,86 & 22,76 & 29,61 & 6,02 \\
\hline
\end{tabular}

Cada estrategia online de campaña se orientó hacia la involucración de las audiencias seleccionadas. Fruto de la recopilación de los textos de las webs, pudo verificarse que los mensajes de las campañas de comunicación organizacional intentaban captar la atención de los responsables de la UE (27\%), los empleados de la EMA (18\%) o el público general (55\%). Aunque la última fue la audiencia seleccionada más común, se debería profundizar sobre si ésta fue la estrategia más exitosa. De hecho, un análisis detallado con respecto a las estrategias más exitosas, también verificó que aquellas que dirigían su mensaje a los trabajadores de la EMA tuvieron una mayor probabilidad de éxito en el proceso de reubicación. 


\subsection{Estrategias más exitosas}

En resumen, nuestra base de datos reveló una serie de tendencias y patrones con respecto hasta qué punto las diferentes estrategias organizacionales online de las ciudades se usaron para atraer votos y convencer a las autoridades de la UE. Entre ellas, nos centraremos en las más exitosas, que pasaron el primer corte.

\section{Amsterdam}

El ganador final, Amsterdam, usó una extensa estrategia online en términos de:

- longitud de texto (5.397 palabras, el cuarto más largo y un $41 \%$ mayor que el promedio);

- vídeos (4 minutos y 55 segundos, el cuarto más largo y un 51\% mayor que el promedio);

- fotos (46, el segundo más extenso y un $54 \%$ mayor que el promedio);

- otros recursos visuales (17, la mayor y un $365 \%$ mayor que el promedio).

La estrategia siguió un acercamiento político/administrativo enfocado hacia temas territoriales y el cumplimiento con los criterios requeridos. Además, la estrategia del candidato apuntó principalmente a los responsables de la UE, a los empleados de la EMA y a sus familias. El apoyo directo de los actores en el sitio online debe destacarse, con hasta ocho testimonios, que principalmente fueron políticos y funcionarios públicos (3,13\% del texto), así como académicos e investigadores (1,26\% del texto). De hecho, el Primer Ministro holandés, Mark de Rutte, lideró el proceso.

En cuanto al contenido y a la selección de las temáticas, Amsterdam demostró una estrategia extensa basada en:

- presentación del lugar

- accesibilidad y ubicación

- sector sanitario

- calidad de vida

- comunidades expatriadas

- capacidades para la transición de la reubicación.

Con la ayuda de NVivo 12 Plus verificamos cómo, cuantitativamente, Amsterdam enfatizó mayormente:

- calidad de vida $(29,67 \%$

- comunidades expatriadas (25,65\%)

- capacidades para la transición de la reubicación (24,06\%)

- accesibilidad y ubicación (19,48\%).

Finalmente, la ciudad candidata cumplió cada criterio del Consejo, exceptuando el último (distribución geográfica).

\section{Milán}

Perdiendo finalmente por una moneda al aire, pero siendo la ganadora en la primera ronda con 25 votos, Milán exhibió una estrategia online por encima de la media en términos de:

- longitud de texto (4.848 palabras, la quinta más larga y un $26,5 \%$ más que el promedio);

- vídeos (2 minutos y 38 segundos, la séptima más reducida y un 19\% menos de extensión que el promedio);

- fotos (32, la sexta más extensa y un 7\% más que el promedio);

- otros recursos visuales (6, la quinta más extensa y un $22 \%$ más que el promedio).

La estrategia siguió un planteamiento político/administrativo, basado en temas territoriales y en el cumplimiento con los criterios. Además, la estrategia de la candidata se enfocó mayormente en los responsables de la UE y el público general. La falta de apoyo directo de los diferentes actores debe destacarse, sin ningún tipo de apoyo (ni siquiera político) revelado.

En cuanto a las temáticas preferentes utilizadas por la campaña de comunicación online, Milán siguió una extensa estrategia enfocada en:

- presentación del lugar

- accesibilidad y ubicación

- sector sanitario

- calidad de vida

- comunidades expatriadas

- opciones de inversión y negocios.

La capital lombarda apostó primero por

- calidad de vida (26,76\%)

- sector sanitario (18,57\%)

- comunidades expatriadas $(17,44 \%)$

- accesibilidad y ubicación (17,31\%).

Finalmente, la candidata cumplió cada criterio excepto el último (distribución geográfica). 


\section{Copenhague}

La tercera ciudad candidata más exitosa (pasó la primera ronda con 20 votos, pero no la segunda) tuvo, en general, una estrategia mucho más limitada en términos de:

- longitud de texto (151 palabras, la más reducida y un 96\% menos que el promedio);

- vídeos ( 2 minutos y 52 segundos, la octava más baja y un $12 \%$ menos que el promedio);

- fotografías (1, la menor y un 96,6\% menos que el promedio);

- otros recursos visuales (5, la séptima más extensa y un $3 \%$ más que el promedio).

La estrategia siguió un planteamiento meramente político/administrativo basándose en temas territoriales y el cumplimiento de los criterios. Además, la estrategia de la candidata se enfocó preferentemente al público general. Debería reconocerse la falta de apoyo directo de los actores al sitio online, con excepción del importante pero único testimonio del Primer Ministro danés, Lars Lokke, en el vídeo vinculado, pero no en el texto online.

En cuanto a la selección temática, Copenhague siguió una estrategia regular en términos de extensión, enfocándose en:

- accesibilidad y ubicación

- sector sanitario

- comunidades expatriadas

- negocios y oportunidades de inversión.

De esta manera, puso el foco principalmente en las

- comunidades expatriadas $(23,22 \%)$

- sector sanitario $(15,64 \%)$

- accesibilidad y ubicación (13,34\%)

- negocios y oportunidades de inversión (11,34\%).

\section{Discusión y conclusiones}

Esta investigación analiza y disecciona las campañas políticas e institucionales online de las ciudades candidatas a la European Medicines Agency (EMA) y destaca los encuadres y las variables en discusión durante el proceso de selección y dentro del contexto de incertidumbre que rodea al Brexit (Bouza-García, 2017; Patel; Reh, 2016; Tuñón-Navarro; Carral, 2018). Con respecto a la principal pregunta de investigación o hipótesis del estudio, nos centramos en verificar si había habido un patrón común entre las estrategias online de las candidatas más exitosas en el proceso de reubicación de la EMA, concretamente si las ciudades candidatas más exitosas presentaron una estrategia online más destacada (en términos de cantidad y calidad) que las otras ciudades candidatas. Por consiguiente, buscamos aplicar los llamados modelos resource push o resource pull (es decir si la posesión de recursos o la intención de obtenerlos condicionaron la extensión de las campañas institucionales online) (Jones; Keating, 1995; Rhodes, 1995; Tuñón-Navarro, 2009) o identificar la falta de aplicabilidad de aquellas (Tatham, 2010; Tatham; Bauer, 2014).

Encontramos algunas características o patrones relevantes. Se pudo constatar que los candidatos exitosos (aquellos que superaron la primera ronda) o al menos aquellos con más votos, invirtieron mayores esfuerzos en sus campañas online. Esto fue cierto, tanto para Amsterdam como para Milán, pero no en el caso de Copenhague, que presentó una estrategia más limitada, en términos de extensión. Además, entre las ciudades no finalistas, algunas llevaron a cabo campañas extensas con buenos resultados, aunque finalmente no pasaron el corte (Atenas o Estocolmo), mientras que la estrategia más extensa de todas (Lille), no obtuvo resultados satisfactorios. Asumimos, entre otras, que la influencia de otras variables de tipo político, relacionadas con la reubicación de la European Banking Authority (EBA) a París, redujeron considerablemente las posibilidades de Lille para albergar la EMA.

En relación con el contenido de la comunicación organizacional, también encontramos algunos hallazgos relevantes. El análisis destacó algunas palabras clave que podían representar el contenido de las estrategias online de las candidatas. De hecho, se detectó un tipo de patrón común entre las ciudades candidatas más y menos exitosas, ya que tuvieron un índice de coincidencia de hasta un $65 \%$ en las 20 palabras más frecuentes (EMA, europeo, ciudad, internacional, ofrece, agencia, mundo, salud, área, nuevo, escuela, Europa y ubicación). Por consiguiente, los temas principales no podían predecir por sí solos un candidato exitoso. Al tratarse de una campaña de comunicación política institucional, pero que a la vez debía adherirse a un pliego de condiciones, se verificó una gran homogeneidad de contenidos y coincidencia incluso de palabras más frecuentes. Por ese motivo, no supuso una variable, precisamente decisiva, en cuanto al resultado final del proceso.

También en relación con el contenido, debería destacarse un acercamiento político/administrativo, pero no científico. De hecho, las palabras más frecuentes relacionadas con la salud o la ciencia fueron escasas (investigación, medicina y
Albergar la EMA era considerado una joya para cualquiera de las 19 ciudades candidatas a suceder a Londres, asumiendo una plantilla de 900 personas con sus respectivas familias, un presupuesto de 320 millones de euros al año, y la tarea de evaluar y autorizar la comercialización de nuevas medicinas en la UE 
salud para las menos exitosas, y salud para las más exitosas). Se pudo detectar caso por caso para las más exitosas (Amsterdam, Milán y Copenhague) un enfoque más autorreferencial que descriptivo de contenidos, lo que nos hace pensar que fueron redactados por especialistas en comunicación, más que por técnicos en materia científica.

Con respecto a la selección temática y el cumplimiento de los criterios, argumentamos que:

- era posible verificar una lógica o relación entre los temas preferentes usados por los candidatos más exitosos en función de la variedad y/o la definición de los mismos;

- tanto los candidatos más como los menos exitosos cumplieron los criterios requeridos de una manera homogénea en sus estrategias online, sin ser esto un elemento diferenciador.

Así pues, pudimos detectar que, por mucho que el sistema de elección derivara del éxito y del impacto de las campañas institucionales sobre los propios estados que debían decidir en el Consejo de la Unión Europea, en relación con la selección temática, se verificó como elemento diferenciador la atención prestada en primer lugar a la calidad de vida en la nueva ciudad, como tema más frecuente y atractivo entre las ciudades candidatas. Seguidamente, el sector sanitario y la presentación del lugar constituyeron las segundas temáticas textuales más frecuentes, en las estrategias de comunicación organizacional. Dichas aproximaciones no son solamente conclusiones aisladas de nuestro estudio, sino que refrendan recientes trabajos en la materia (Trendafilova et al., 2019).

Asimismo, se pudo verificar que las estrategias online de las ciudades candidatas difirieron en términos de extensión y prioridades. Se detectó un tipo de correlación entre aquellas estrategias que enmarcaron más temas y el nivel final de éxito. De hecho, Amsterdam y Milán encuadraron seis categorías, mientras que el promedio global fue sólo de cuatro. Copenhague (la medalla de bronce) sólo encuadró cuatro temas (promedio), opción más común entre los candidatos menos exitosos. Sin embargo, la variedad y extensión del encuadre constituyó una precondición para el éxito, pero no lo garantizó. De hecho, los candidatos menos exitosos encuadraron menos categorías en general, aunque Zagreb (un candidato no exitoso) encuadró seis temas, el mismo número que las exitosas Amsterdam y Milán.

Los resultados anteriores se respaldaron parcialmente para los candidatos más exitosos (Amsterdam, Milán y Copenhague). Con la ayuda de un software identificamos el promedio de extensión de los textos online dedicados a las diferentes selecciones temáticas. De hecho, las comunidades expatriadas se mostraron más relevantes que la calidad, accesibilidad y ubicación, y los sectores sanitarios, a la hora de ser los encuadres principales a los que los candidatos exitosos prestaron atención. En comparación con datos anteriores, notamos que aunque los candidatos se enfocaron generalmente en la calidad de vida, el sector sanitario y la presentación del lugar, los ganadores sustituyeron la presentación del lugar por las comunidades expatriadas como variable clave explicativa de su éxito. Además, los candidatos más y menos exitosos cumplieron los criterios requeridos de una manera homogénea en sus campañas online, sin ser éste un elemento diferenciador.

Con respecto a los actores y las audiencias, argumentamos también que: la variedad, el número y el perfil político de los actores representados en las estrategias online favorecerían el éxito de las candidatas y que las más exitosas debían ser aquellas que dirigieran el mensaje preferentemente a las elites europeas.

En primer lugar, descubrimos que hasta un $77 \%$ de las campañas online involucraron a políticos o funcionarios públicos, seguidos de académicos (15\%) y finalmente hombres de negocios (8\%). Esta dominancia del uso de políticos como fuentes de información en comunicación de temas sobre salud es una realidad que ha sido descrita en estudios previos (Catalán-Matamoros; Peñafiel-Saiz, 2019; Deprez; Van-Leuven, 2017). Además, detectamos claras divergencias en relación con esta variable, ya que la mitad de los candidatos optaron por introducir actores para apoyar sus campañas online (Amsterdam, Atenas, Bonn, Bratislava, Bucarest, Copenhague, Lille y Estocolmo), mientras que la otra mitad no lo consideró beneficioso. Entre los seis candidatos que dieron voz a los actores en sus textos de campaña online, sólo Amsterdam pasó la primera ronda. Por consiguiente, no se puede inferir que los candidatos más exitosos introdujeron más actores, ya que Milán y Copenhague presentaron estrategias muy limitadas en esos términos. Sin embargo, el software sirvió como intercoder para verificar la prominencia de los políticos y los funcionarios públicos como actores en cada caso, excepto Lille, cuya candidatura fue principalmente apoyada por sus investigadores y académicos. Por consiguiente, la sub-hipótesis de los actores no ha sido probada convenientemente. La variedad y el número de los actores que apoyan a un candidato demostró no ser un factor decisivo que determinara el éxito. De hecho, la mitad de los candidatos ni siquiera los incluyeron. Los políticos y los funcionarios públicos fueron los actores más presentes, pero no fue posible detectar una correlación clara entre su presencia y el éxito de sus candidatas.

Nuestros hallazgos verificaron que las estrategias organizacionales online de los candidatos tendían a enfocarse al público general (Campos-Domínguez, 2017; López-Meri; Marcos-García; Casero-Ripollés, 2017) más que en los responsables de la UE o los empleados de la EMA. Sin embargo, esto no demostró ser una estrategia efectiva, porque si ahondamos en el análisis, podemos verificar que los candidatos más exitosos, prefirieron centrarse en las co-
Los candidatos más exitosos fueron los que dirigieron el mensaje preferentemente a la UE y a los empleados de la EMA 
munidades expatriadas y por tanto en los empleados de la EMA. Por consiguiente, y confirmando la subhipótesis que concierne a las audiencias, así como a la investigación previa (Lauristin, 2007; Mancini; Mazzoni, 2015), las estrategias que se enfocaron en las élites europeas (empleados de la $E M A$ ) fueron más propensas a tener éxito en el proceso de reubicación. Se coincide, por tanto, con algunas recientes investigaciones, que han demostrado la tendencia que existe en el contexto europeo de dirigirse primordial y en ocasiones casi exclusivamente a las propias élites europeas, diseñando así procesos de comunicación eminentemente introspectivos (Tuñón; Carral, 2019).

En resumen, pese a no poder hacer generalizaciones rotundas sobre cuáles son las claves del éxito de una estrategia de city-branding, de un modo deductivo, se han podido encontrar ciertos patrones comunes entre las candidaturas seleccionadas para última ronda (Amsterdam y Milán) que explican su éxito en contraposición a la eliminada en segunda votación (Copenhague). Asimismo, la comparación simultánea que se puede realizar entre las dos finalistas también muestra cuáles hubieran sido los motivos principales por los cuales el jurado podría haberse decantado por la capital holandesa de manera objetiva, en caso de no haber optado por el lanzamiento de una moneda.

Tabla 6. Comparativa de características de las candidaturas finalistas

\begin{tabular}{|c|c|c|c|}
\hline & \multirow[b]{2}{*}{ Amsterdam } & \\
\hline & & Milán & Copenhague \\
\hline $\begin{array}{l}\text { Extensión textual y del contenido } \\
\text { audiovisual }\end{array}$ & $\begin{array}{l}\text { Entre las candidaturas con mayor } \\
\text { contenido y con un } 50 \% \text { más de } \\
\text { promedio que la media general }\end{array}$ & $\begin{array}{l}\text { Entre las candidaturas posicio- } \\
\text { nadas en la mitad de la tabla sin } \\
\text { superar el promedio }\end{array}$ & $\begin{array}{l}\text { Entre las candidaturas con menor } \\
\text { contenido }\end{array}$ \\
\hline Repetición textual & $\begin{array}{l}\text { Gran repetición de ciertas pala- } \\
\text { bras que ayuda a recalcar un dis- } \\
\text { curso claro y conciso. La mayoría } \\
\text { reiteradas entre } 20 \text { y } 60 \text { veces }\end{array}$ & $\begin{array}{l}\text { Gran repetición de ciertas pala- } \\
\text { bras. La mayoría de reiteraciones } \\
\text { se suceden entre las } 15 \text { y } 45 \\
\text { veces. }\end{array}$ & $\begin{array}{l}\text { Bajo número de reiteraciones y } \\
\text { poca repetición de las mismas. } \\
\text { Ninguna palabra se reproduce en } \\
5 \text { ocasiones }\end{array}$ \\
\hline Número de temas & 6 & 6 & 4 \\
\hline Audiencia & Empleados de EMA & Empleados de EMA & Público en general \\
\hline $\begin{array}{l}\text { Utilización de temas según au- } \\
\text { diencia clave (empleados) }\end{array}$ & $\begin{array}{l}\text { 4/4, obteniendo el porcentaje } \\
\text { más alto en todas ellas }\end{array}$ & $3 / 4$ & $2 / 4$ \\
\hline Inclusión de actores & Sí & No & $\begin{array}{l}\text { No (sólo en el vídeo, pero no en } \\
\text { el texto) }\end{array}$ \\
\hline
\end{tabular}

En base a la tabla 6 se puede observar claramente el porqué de la exclusión de Copenhague en la última ronda, puesto que no cumple los requisitos de forma tan brillante como lo hacen Amsterdam y Milán. Su presentación se muestra pobre en cuanto a contenido y reiteración de un discurso conciso, además de no atender a todos los temas propuestos ni de ampliar la tipología de actores. Igualmente, la candidatura se decanta por dirigirse a un público general, como lo hacen la mayoría de las ciudades que se presentan, no obstante, se ha demostrado que el verdadero éxito residía en hablar a los círculos europeos (Lauristin, 2007; Mancini; Mazzoni, 2015). Por ello, la exposición danesa no supo abordar completamente (tan sólo 2 de 4) las temáticas desde la perspectiva más interesante para la audiencia clave (los empleados de la EMA).

Llegados a la votación final, de no ser el azar quien hubiera elegido a la ganadora, la elección de la candidatura óptima se habría decidido en función de pequeños detalles. Si bien ambas candidatas, Amsterdam y Milán, acertaron a la hora de dirigirse a un público específico al que presentaron un amplio abanico de hasta 6 temas, fue la capital holandesa quien se esmeró de manera más profunda en su exposición. Como muestra la tabla 6, tanto en la extensión de su contenido (imagen y texto) como en su explicación, Amsterdam supo formular su discurso por encima de la media de ciudades (incluida Milán), y fue más precisa en su selección temática, segmentando y priorizando su audiencia preferente, los empleados de la EMA. De hecho, atendiendo a las cuatro cuestiones que podrían suscitar mayor preocupación (accesibilidad/localización de su nueva ciudad; calidad de vida; reubicación; comunidad de expatriados) entre los interesados, Amsterdam mencionó cuatro de cuatro, uno más que Milán (3/4), además de profundizar de manera más extensa en todos ellos (tabla 4).

Finalmente, queremos también apuntar las limitaciones de este estudio. No se trata de un proceso de campaña tradicional por web en un análisis de contexto de elecciones (Dimitrova et al., 2014; Lilleker et al., 2011; Casero-Ripollés; Feenstra; Tormey, 2016; López-García, 2016). En cambio, la investigación se ha enfocado en el branding online de las ciudades candidatas en el proceso de reubicación de la EMA a través de sus portales web ad hoc. Por consiguiente, su énfasis primario radica en la comunicación organizacional online y concretamente en el contenido del texto. Se podrían haber producido diferentes resultados si se hubiese elegido un enfoque alternativo. A modo de ejemplo, uno eminentemente cualitativo y que hubiera profundizado en la personalización de las campañas (López-Meri; Marcos-García; Casero-Ripollés, 2017; o Bouza-García; Tuñón-Navarro, 2018) de cada una de las ciudades candidatas a través de figuras políticas relevantes que bien hubieran liderado o al menos contribuido a la campaña organizacional, tal y como sucedió en los casos de los primeros ministros holandés (Mark de Rutte) y danés (Lars Lokke). De hecho, aunque el análisis se basó primariamente en el

Este estudio hace una contribución importante al campo de las campañas organizacionales online, así como aporta una metodología para el análisis de contenido online relacionado con la UE, las etnografías digitales y la comunicación política/institucional 
contenido del texto, recogimos también algunos elementos visuales incluyendo imágenes y videos, que podrían usarse para un futuro análisis a fondo e investigación. Además, la especificidad del contexto político del proceso de reubicación no facilitará un seguimiento del análisis en el futuro, ya que las reubicaciones de agencias europeas son excepcionales.

Sin embargo, los descubrimientos de nuestra investigación, no sólo vienen a refrendar recientes trabajos que han abordado de manera pionera el análisis (descriptivo) de las campañas web de las ciudades candidatas a la reubicación de la EMA (Trendafilova et al., 2019), u otros acerca de la dominancia del contenido político en la campañas de comunicación en materia de salud (Catalán-Matamoros; Peñafiel-Saiz, 2019; Deprez; Van-Leuven, 2017), sino que algunas de sus observaciones suponen una contribución en un doble ámbito de la bibliografía científica:

- en el de los trabajos sobre campañas de comunicación y branding de ciudades (Zapata-Garesché, 2007; Van-der-Pluijm; Melissen, 2007; Kavaratzis; Ashworth, 2010; o Lara-Pacheco, 2015; entre otros);

- creemos que nuestro estudio provee datos originales relevantes y hace una contribución específica importante al campo incipiente de las campañas organizacionales online en la zona pan-europea (Quittkat, 2011; Davesa; Shahin, 2014; Papagianneas, 2017; o Bouza-García; Oleart; Álvaro; Tuñón-Navarro, 2019; entre otros).

Por todo lo anterior, este trabajo supone igualmente una contribución metodológica, desde el análisis de contenido (aproximación eminentemente cuantitativa) relacionado con la UE, concretamente al marco de las campañas organizacionales online.

\section{Referencias}

Aalberg, Toril; Strömbäck, Jesper; De-Vreese, Claes H. (2012). "The framing of politics as strategy and game: A review of concepts, operationalizations and key findings". Journalism, v. 13, n. 2, pp. 162-178.

https://doi.org/10.1177/1464884911427799

Amendola, Giandomenico (2000). La ciudad postmoderna. Magia y miedo de la metrópolis contemporánea. Madrid: Celeste Ediciones. ISBN: 8482112392

https://leerlaciudadblog.files.wordpress.com/2019/02/amendola-la-ciudad-postmoderna.pdf

Arroyo-Almaraz, Isidoro; Calle-Mendoza, Samuel; Van-Wyk, Cliff (2018): “La eficacia en la comunicación de las ONG. El uso de Facebook en campañas de emergencia”. Revista latina de comunicación social, n. 73, pp. 765-789.

http://www.revistalatinacs.org/073paper/1281/40es.html

Bardin, Laurence (2002). Análisis de contenido. Madrid: Akal. ISBN: 9788476000939

Bouza-García, Luis (2017). "The New Narrative Project and the politicisation of the EU”. Journal of contemporary European studies, v. 25, n. 3, pp. 340-353.

https://doi.org/10.1080/14782804.2017.1348340

Bouza-García, Luis; Oleart, Álvaro; Tuñón-Navarro, Jorge (2019). “Framing EU trade policy online: The case of @NoAlTTIP on Twitter". Communication \& society, v. 32, n. 4, pp. 257-273.

https://doi.org/10.15581/003.32.4.257-273

Bouza-García, Luis; Tuñón-Navarro, Jorge (2018). “Personalización, distribución, impacto y recepción en Twitter del discurso de Macron ante el Parlamento Europeo el 17/04/18”. El profesional de la información, v. 27, n. 6, pp. 1239-1247. https://doi.org/10.3145/epi.2018.nov.07

Campillo-Alhama, Concepción; Martínez-Sala, Alba-María (2017). "Integrated communication 2.0 in municipal administration". El profesional de la información, v. 26, n. 3, pp. 507-515.

https://doi.org/10.3145/epi.2017.may.17

Campos-Domínguez, Eva (2017). “Twitter y la comunicación política”. El profesional de la información, v. 26, n. 5, pp. 785-793. https://doi.org/10.3145/epi.2017.sep.01

Canel, María-José; Sanders, Karen (2012). "Government communication: An emerging field in political communication research". In: Semetko, Holli A.; Scammell, Margaret (eds.). The SAGE handbook of political communication, pp. 85-96. London: SAGE. https://mariajosecanel.com/pdf/emergingfield.pdf https://doi.org/10.4135/9781446201015.n8

Casero-Ripollés, Andreu; Feenstra, Ramón A.; Tormey, Simón (2016). "Old and new media logics in an electoral campaign: The case of Podemos and the two-way street mediatization of politics". The international journal of press/politics, v. 21, n. 3, pp. 378-397. https://doi.org/10.1177/1940161216645340

Catalán-Matamoros, Daniel; Peñafiel-Saiz, Carmen (2019). "Specialty matters. Analysis of health journalists' coverage about vaccines". El profesional de la información, v. 28, n. 2, e280201.

https://doi.org/10.3145/epi.2019.mar.01

Costa-Sánchez, Carmen (2014). "Transmedia storytelling, an ally of corporate communication: \#Dropped by Heineken case study". Communication \& society, v. 27, n. 3, pp. 127-150.

https://revistas.unav.edu/index.php/communication-and-society/article/view/35992 
Cusin, François; Damon, Julien (2012). "La atractividad, condición de las ciudades del futuro. Clasificaciones, retos y estrategias urbanas". Sotavento, n. 18, pp. 80-89.

https://dialnet.unirioja.es/servlet/articulo?codigo $=5137606$

D’Adamo, Orlando; García-Beaudox, Virginia (2016). “Comunicación política: narración de historias, construcción de relatos políticos y persuasión". Comunicación y hombre, n. 12, pp. 23-39.

http://www.redalyc.org/articulo.oa?id=129446703001

Davesa, Ferrán; Shahin, Jamal (2014). "Evaluating Euro-parties' use of digital tools for the 2014 EP election campaign". In: Spring conference elections and democracy in Europe, 7-9 April. Brussels, Belgium.

Deprez, Annelore; Van-Leuven, Sarah (2017). "About pseudo quarrels and trustworthiness". Journalism studies, v. 19, n. 9, pp. 1257-1274.

https://doi.org/10.1080/1461670X.2016.1266910

Dimitrova, Daniela V.; Shehata, Adam; Strömbäck, Jesper; Nord, Lars W. (2014). "The effects of digital media on political knowledge and participation in election campaigns". Communication research, v. 41, n. 1, pp. 95-118.

https://doi.org/10.1177/0093650211426004

Eom, Young-Ho; Puliga, Michelangelo; Smailović, Jasmina; Mozetič, Igor; Caldarelli, Guido (2015). "Twitter-based analysis of the dynamics of collective attention to political parties". PLoS one, v. 10, n. 7.

https://doi.org/10.1371/journal.pone.0131184

Fernández-Falero, María-Rosario; Trabadela-Robles, Javier; Garcés-Botacio, Indhira; Ruano-López, Soledad (2017). "Comunicación política de los ayuntamientos a través de sus webs. Caso de Extremadura". El profesional de la información, v. 26, n. 3, pp. 404-410.

https://doi.org/10.3145/epi.2017.may.06

García-Orosa, Berta (2009). Gabinetes de comunicación online. Sevilla: Comunicación Social. ISBN: 9788496082786

García-Orosa, Berta; Vázquez-Sande, Pablo; López-García, Xosé (2017). “Narrativas digitales de los principales partidos políticos de España, Francia, Portugal y Estados Unidos". El profesional de la información, v. 26, n. 4, pp. 589-600. https://doi.org/10.3145/epi.2017.jul.03

Guerrero-Pico, Mar; Scolari, Carlos A. (2016). "Narrativas transmedia y contenidos generados por los usuarios: el caso de los crossovers". Cuadernos.info, n. 8, pp. 183-200.

https://doi.org/10.7764/cdi.38.760

Gutiérrez-Rubí, Antoni (2014). Tecnopolítica. Madrid: Bebookness. ISBN: 9788469718063

http://www.gutierrez-rubi.es/tecnopolitica

Habermas, Jürgen (2017). “¿Qué nos costará esta vez a los alemanes?”. El país, 10 diciembre. https://elpais.com/elpais/2017/12/08/opinion/1512750567 704832.html

Hatem, Fabrice (2004). “Attractivité: de quoi parlons nous?”. Pouvoirs locaux, v. 61. http://www.fabricehatem.fr/fh-medias/publications/attractivit_pl.pdf

lyengar, Shanto; Simon, Adam F. (2000). "New perspectives and evidence on political communication and campaign effects". Annual review of psychology, v. 51, pp.149-169.

https://doi.org/10.1146/annurev.psych.51.1.149

Jones, Barry; Keating, Michael (eds.) (1995). The European Union and the regions. Oxford: Clarendon Press. ISBN: 978 0198279990

https://doi.org/10.1093/019827999X.001.0001

Kavaratzis, Mihalis; Ashworth, Gregory (2010). “Place branding: where do we stand?". In: Ashworth, Gregory; Kavaratzis, Mihalis. Towards effective place brand management. Branding European cities and regions. Cheltenham-Northhampton: Edward Elgar. ISBN: 9781848442429

Kotler, Philip; Armstrong, Gary (2001). Fundamentos de marketing. Ciudad de México: Pearson Educación. ISBN: 978 9702611868

Kreiss, Daniel (2016). "Seizing the moment: The presidential campaigns' use of Twitter during the 2012 electoral cycle". New media \& society, v. 18, n. 8, pp. 1473-1490. https://doi.org/10.1177/1461444814562445

Lara-Pacheco, Ray-Freddy (2015). La construcción de las ciudades modelo y su inserción internacional. Tesis doctoral. Universidad del País Vasco.

https://bit.ly/3ePnO58 
Larrondo-Ureta, Ainara (2016). “Comunicación organizacional ante el reto de la estrategia multiplataforma y 2.0: la experiencia de los partidos políticos en el País Vasco". El profesional de la información, v. 25, n. 1, pp. 114-123. https://doi.org/10.3145/epi.2016.ene.11

Larsson, Anders O.; Kalsnes, Bente (2014). “'Of course we are on Facebook': Use and non-use of social media among Swedish and Norwegian politicians". European journal of communication, v. 29, n. 6, pp. 653-667. https://doi.org/10.1177/0267323114531383

Lauristin, Marju (2007). "The European public sphere and the social imaginary of the 'new Europe'”. European journal of communication, v. 22, n. 4, pp. 397-412.

https://doi.org/10.1177/0267323107083058

Lilleker, Darren G.; Koc-Michalska, Karolina; Schweitzer, Eva J.; Jakunski, Michal; Jackson, Nigel; Vedel, Thierry (2011). Informing, engaging, mobilizing or interacting: Searching for a European model of web campaigning. European journal of communication, v. 26, n. 3, pp. 195-213.

https://doi.org/10.1177\%2F0267323111416182

Llaudes, Salvador; Molina, Ignacio; Otero-Iglesias, Miguel; Steinberg, Federico (2018). Spain and the prospect of Brexit. Elcano Policy Paper 3/2018 - 15/6/2018.

https://bit.ly/2KPEEI7

López-Alonso, Esmeralda; Moreno-López, Begoña (2019). “La gestión de los medios sociales en la Administración local: análisis de los municipios rurales españoles". Vivat academia, n. 148, pp. 77-99.

https://doi.org/10.15178/va.2019.148.77-99

López-García, Guillermo (2016). “'New' vs 'old' leaderships: The campaign of Spanish general elections 2015 on Twitter". Comunicación y sociedad, v. 29, n. 3, pp. 149-168.

https://doi.org/10.15581/003.29.3.149-168

López-Meri, Amparo; Marcos-García, Silvia; Casero-Ripollés, Andreu (2017). "What do politicians do on Twitter? Functions and communication strategies in the Spanish electoral campaign of 2016". El profesional de la información, v. 26, n. 5, pp. 795-804.

https://doi.org/10.3145/epi.2017.sep.02

Maarek, Philippe J. (2014). "Politics 2.0: New forms of digital political marketing and political communication". Trípodos, n. 34, pp. 13-22.

http://www.tripodos.com/index.php/Facultat_Comunicacio_Blanquerna/article/view/163

Mancini, Paolo; Mazzoni, Marco (2015). “Countries still matter". In: Picard, Robert G. (ed.). The Euro crisis in the media. Oxford: Tauris and Reuters Institute for the Study of Journalism, pp. 177-194. ISBN: 9781784530594 https://doi.org/10.5040/9780755694990.ch-009

Marfil-Carmona, Rafael (2013). "Interactividad digital y estrategias narrativas en la publicidad audiovisual de Manos Unidas y Unicef”. Historia y comunicación social, v. 18, pp. 169-181. https://doi.org/10.5209/rev_HICS.2013.v18.43957

Martínez-Valero, Laura (2016). “Narrativas transmedia en la comunicación de las ONG. Sí me importa de Oxfam Intermón". Fonseca, journal of communication, n. 13, pp. 33-53.

https://doi.org/10.14201/fjc201613354

Papagianneas, Stavros (2017). Rebranding Europe. Fundamentals for leadership communication. Brussels: ASP editions. ISBN: 9789057186202

Patel, Oliver; Reh, Christine (2016). Brexit: The consequences for the EU's political system. UCL Constitution Unit Briefing Paper.

https://www.ucl.ac.uk/european-institute/news/2016/may/brexit-consequences-eus-political-system

Peñafiel, Carmen (2016). "Reinvención del periodismo en el ecosistema digital y narrativas transmedia". AdComunica, n. 12 , pp. 163-183.

https://doi.org/10.6035/2174-0992.2016.12.10

Quittkat, Christine (2011). "The European Commission's online consultations: A success story?". Journal of common market studies, v. 44, n. 3, pp. 653-674.

https://doi.org/10.1111/j.1468-5965.2010.02147.x

Rhodes, Martin (ed.) (1995). The regions and the new Europe: Patterns in core and periphery development. Manchester: Manchester University Press. ISBN: 9780719042515 
Salaverría, Ramón (2015). “Los labs como fórmula de innovación en los medios”. El profesional de la información, v. 24, n. 4, pp. 397-404. https://doi.org/10.3145/epi.2015.jul.06

Sánchez-Murillo, Luis-Fernando (2005). "El marketing político y sus consecuencias para la democracia”. Comunicación y sociedad, n. 4, pp. 11-38.

http://www.comunicacionysociedad.cucsh.udg.mx/index.php/comsoc/article/view/4091

Scolari, Carlos A.; Fernández-de-Azcárate, Sara; Garín, Manuel; Guerrero, Mar; Jiménez, Manel; Martos, Aitor; Obradors, Matilde; Oliva, Mercè; Pérez, Óliver; Pujadas, Eva (2012). “Narrativas transmediáticas, convergencia audiovisual y nuevas estrategias de comunicación". Quaderns del CAC, v. 15, n. 1, pp. 79-89.

https://www.cac.cat/sites/default/files/2019-01/Q38_scolari_et_al_ES.pdf

Semetko, Holli A.; De-Vreese, Claes H. (2004). Political campaigning in referendums: Framing the referendum issue. Abingdon: Routledge. ISBN: 9780415349413

Serrano-Cobos, Jorge (2016). "Tendencias tecnológicas en internet: hacia un cambio de paradigma". El profesional de la información, v. 25, n. 6, pp. 843-850.

https://doi.org/10.3145/epi.2016.nov.01

Tatham, Michaël (2010). "'With or without you'? Revisiting territorial state-bypassing in EU interest representation”. Journal of European public policy, v. 17, n. 1, pp. 76-99.

https://doi.org/10.1080/13501760903465082

Tatham, Michaël; Bauer, Michael W. (2014). "Competence ring-fencing from below? The drivers of regional demands for control over upwards dispersion". Journal of European public policy, v. 21, n. 9, pp. 1367-1385.

https://doi.org/10.1080/13501763.2014.923017

Trendafilova, Petya; Benisheva, Tatyana; Sidjimova, Dobriana; Polyakova, Bilyana (2019). “Brexit consequences: Relocation of the European Medicines Agency". Scripta scientifica pharmaceutica, v. 6, n. 1, pp. 16-25.

http://journals.mu-varna.bg/index.php/ssp/article/view/5976

Túñez-López, Miguel (2012). La gestión de la comunicación en las organizaciones. Sevilla: Comunicación Social Ediciones y Publicaciones. ISBN: 9788492860937

Tuñón-Navarro, Jorge (2009). La activación europea de las regiones legislativas (Análisis comparado de las estrategias de Canarias, Escocia, Toscana y Valonia). Tesis doctoral. Universidad Complutense de Madrid.

http://eprints.ucm.es/8715/1/T30787.pdf

Tuñón-Navarro, Jorge (2017). Comunicación internacional. Información y desinformación global en el siglo XXI. Madrid: Fragua. ISBN: 9788470747472

Tuñón-Navarro, Jorge; Bouza-García, Luis; Carral, Uxía (2019). Comunicación Europea ¿A quién doy like para hablar con Europa? Dykinson, Madrid. ISBN: 9788491489771

Tuñón-Navarro, Jorge; Carral, Uxía (2018). Últimas noticias sobre el Brexit económico. Informe del OBS Business School Press, Barcelona.

https://comunicacioneuropea.files.wordpress.com/2018/08/obs.pdf

Tuñón-Navarro, Jorge; Carral, Uxía (2019). “Twitter como solución a la comunicación europea. Análisis comparado en Alemania, Reino Unido y España". Revista latina de comunicación social, v. 74, pp. 1219-1234.

https://doi.org/10.4185/RLCS-2019-1380

Unión Europea (2017). Procedimiento conducente a una decisión sobre la reubicación de la Agencia Europea de Medicamentos y de la Autoridad Bancaria Europea en el contexto de la retirada del Reino Unido de la Unión Europea. Bruselas, 22 junio.

https://www.consilium.europa.eu/media/21503/22-euco-conclusions-agencies-relocation.pdf

Van-der-Pluijm, Rogier; Melissen, Jan (2007). City diplomacy: The expanding role of cities in international politics. La Haya: Netherlands Institute of International Relations Clingendael.

https://www.uclg.org/sites/default/files/20070400_cdsp_paper_pluijm.pdf

Ward, Stephen (2005). Selling places. The marketing and promotion of towns and cities, 1850-2000. Oxon: Taylor \& Francis. https://doi.org/10.4324/9780203976746

Zapata-Garesché, Eugene D. (2007). Manual práctico para internacionalizar la ciudad. Guía para la acción exterior de los gobiernos locales y la cooperación descentralizada - Unión Europea - América Latina. Volumen 1. Barcelona: Diputación de Barcelona.

https://www.ritimo.org/IMG/pdf/Manual_para_internacionalizar_a_la_ciudad.pdf 